\title{
Análisis de Terminología Clínico Odontológica y su Traducción al Mapudungun
}

\author{
Analysis of Clinical Dental Terminology and its Translation to Mapudungun
}

\author{
Tania Flores*; Jacqueline Caniguan** \& Ramón Fuentes ${ }^{*}$
}

FLORES, T.; CANIGUAN, J. \& FUENTES, R. Análisis de terminología clínico odontológica y su traducción al mapudungun. Int. J. Odontostomat., 10(2):249-254, 2016.

RESUMEN: El uso de la medicina occidental tiene un papel preponderante en la búsqueda de comunicación eficaz con el pueblo mapuche, que se hace efectiva mediante la creación de centros de salud intercultural, sin embargo las brechas lingüísticas siguen siendo una gran problemática comunicacional. Los diccionarios bilingües mapudungun-castellano ofrecen información limitada respecto a los términos necesarios para un correcto diagnóstico odontológico. Lo mismo ocurre con traducciones para algunos conceptos que involucran síntomas y signos clínicos de las enfermedades orales. El objetivo de esta investigación fue determinar si los términos más utilizados en odontología tienen traducción al mapudungun. Se realizó un estudio cualitativo, descriptivo y de corte transversal. Con la ayuda de 10 odontólogos de la red odontológica asistencial de la ciudad de Temuco, se elaboró un listado de 132 términos frecuentemente utilizados en la atención odontológica, se analizó su significado con ayuda de diccionarios mapudungun-castellano y posteriormente con ayuda de una especialista en lengua mapuche. Se determinó que un $28,78 \%$ de los términos tienen significado en la lengua mapuche; un $14,39 \%$ no tienen traducción pero pueden comprenderse desde la lengua; mientras que un $56,81 \%$ no tienen traducción ni pueden comprenderse desde la lengua mapudungun. Un importante número de términos usados en odontología no tienen traducción en mapudungun; y aunque entre el pueblo mapuche la concepción acerca de la salud oral pareciera tener mucha importancia, aun son escasos los términos clínicos que se pueden explicar en base a conceptos mapuches preexistentes. Surge por tanto, la necesidad de generar vías de comunicación certera entre paciente y dentista con la creación de nuevas palabras y conceptos en mapudungun, para obtener herramientas que ayuden a comprender la percepción de los conceptos de salud y enfermedad en el contexto de la cosmovisión del paciente mapuche y contribuir al mejoramiento de la atención odontológica.

PALABRAS CLAVE: dentista, mapuche, creencias, salud oral, indígenas.

\section{INTRODUCCIÓN}

En Chile, el 4,6 \% de la población pertenece a pueblos indígenas, siendo el pueblo mapuche el más numeroso, alcanzando el $87,3 \%$ (CEP, 2002), muchos de los cuales han emigrado a las ciudades (Haughney \& Mariman, 1993), manteniéndose concentraciones de comunidades indígenas en las regiones del Bío-Bío y de la Araucanía. En promedio, el pueblo mapuche accede a 9 años de escolaridad y $11 \%$ se mantiene como analfabeto; similar a la escolaridad general del país de 10 años de escolaridad y 4,3\% de analfabetismo (Departamento de Estudios y Desarrollo de la División de Planificación y Presupuesto del Ministerio de Edu- cación, 2004). Mientras un $70 \%$ se autocalifica como perteneciente al nivel socio económico bajo (CEP) son impactados por fenómenos como la relocalización y la pérdida de sus asentamientos históricos, afectando sus condiciones de vida y haciéndoles actualmente minoritarios en su propio territorio (Díaz Mujica et al., 2004). El efecto de la migración se expresa en la disminución de la población residente en las comunidades rurales, lo que conlleva a un debilitamiento de los mecanismos recíprocos que regulan la red social comunitaria, así como la adquisición de patrones de rechazo a la tradición mapuche, en favor de comporta-

\footnotetext{
* Centro de Investigación en Ciencias Odontológicas (CICO), Departamento Odontología Integral del Adulto, Facultad de Odontología, Universidad de La Frontera, Temuco, Chile.

"Facultad de Educación, Ciencias Sociales y Humanidades. Universidad de La Frontera, Temuco, Chile.
} 
mientos y actitudes ligados a la tradición occidental dominante (Errázuriz, 2006). Al mapuche le afecta todo lo que ocurre en la naturaleza; cuando se rompe el equilibrio natural, las relaciones entre los seres se ven afectadas. Si alguien rompe el equilibrio al transgredir leyes de la naturaleza, sufre las consecuencias de lo que ha provocado. Este desequilibrio se manifiesta en el plano físico o psicológico y es conocido como kutran o enfermedad (Marileo, 2002). La falta de salud no sólo se relaciona con la presencia o ausencia de dolor; el dolor es la maduración de la enfermedad que ha ingresado a la persona. Por tanto, se debe considerar aspectos más allá de la expresión concreta de la enfermedad, poniendo atención a manifestaciones tanto de la persona como del medio que la rodea. En la concepción de la enfermedad, se entremezclan la etiología y la sintomatología. El cuerpo no está dividido en sistemas, por lo cual el diagnóstico y tratamiento considera estos dos aspectos (Ibacache, 2001). En odontología, sigue siendo el dolor una de las primeras causas de consulta, asimismo las enfermedades orales de alta prevalencia, como son la caries y la enfermedad periodontal (MINSAL, 2010). Si bien el uso de la medicina occidental se ha visto potenciado a través de la creación de centros de salud intercultural, las brechas comunicacionales existentes con la población mapuche rural, quienes conservan las raíces de la lengua hablada, generan una gran problemática. En este escenario, para lograr confianza con el paciente, y realizar una correcta anamnesis, examen clínico, diagnóstico y explicar un tratamiento, es indispensable contar con las herramientas lingüísticas necesarias (Díaz et al.). En esta búsqueda de comunicación podemos destacar que dentro de la información que ofrecen los diccionarios comunes, faltan términos para hacer un correcto diagnóstico odontológico en mapudungun. En este artículo se utilizarán dos denominaciones para la lengua, mapudungun: término muy difundido para denominar a la lengua; y mapuchedungun, nominación dada por los hablantes. Ambos vocablos se refieren al nombre del idioma mapuche. Por otro lado tampoco se encuentran traducciones para algunos conceptos que involucran síntomas y signos clínicos de las enfermedades orales.

El objetivo de esta investigación fue revisar si los términos más utilizados en odontología tienen traducción al mapudungun y así obtener herramientas que ayuden a comprender la forma de percibir los conceptos de salud y enfermedad en el contexto de la cosmovisión del paciente mapuche.

\section{MATERIAL Y MÉTODO}

Se realizó un estudio de tipo cualitativo, descriptivo y de corte transversal. Se elaboró un listado de términos utilizados frecuentemente durante la atención odontológica, el que se presentó a 10 odontólogos que se desempeñan en diversos centros de atención primaria en la Región de la Araucanía durante el año 2015, y se les solicitó que identificaran los términos más utilizados por ellos durante la atención clínica de pacientes, conformándose un listado final de 132 términos. Se buscó en diccionarios bilingües castellanomapudungun los significados para los términos de dicho listado (Augusta, 1995, 2007; Antinao Varas, 2014). Se determinó que algunos de estos términos existen en el idioma mapuche, sin embargo otros no. Los vocablos no existentes en la lengua, se analizaron y clasificaron en dos categorías: por un lado, términos que pueden crearse recurriendo a los mecanismos propios de la lengua, por ejemplo cepillo de dientesküchaforopeyüm:

$$
\begin{aligned}
& \text { Kücha-foro-peyüm } \\
& \text { Lavar-diente-INSTRUMENTO } \\
& \text { "cepillo de dientes" }
\end{aligned}
$$

y por otro lado, términos para los cuales se pueden usar préstamos del castellano (un préstamo lingüístico es un fenómeno en el cual una lengua carece de un concepto y toma una palabra de otra lengua para ampliar su repertorio o bien, en muchos casos, para reemplazar una palabra ya existente) dado que el concepto o idea no existe en la lengua original. En consecuencia se dispuso la siguiente clasificación:

1: Existe traducción literal.

2: No existe traducción literal, pero puede comprenderse el concepto desde la lengua.

3: No existe traducción, ni puede comprenderse el concepto.

\section{RESULTADOS}

De los 132 términos frecuentemente utilizados en la atención clínica de pacientes odontológicos, un $28,78 \%$ (38) tienen traducción literal en la lengua mapuche (Tabla I); para un 14,39 \% (19) no existe traducción literal, sin embargo, es posible entenderlos desde el idioma mapuche (por ejemplo: cepillo de dientes- küchaforopeyüm) (Tabla II); mientras que para un 
Tabla I. Listado de términos clínico-odontológicos con traducción literal en lengua mapuche.

\begin{tabular}{|c|c|}
\hline Término odontológico & Traducción Mapuchedungun \\
\hline Blanqueamiento dental & ligdewmantun \\
\hline Boca & wün \\
\hline Caries & Üngoñ \\
\hline Colutorios & küchatuwe \\
\hline Diente & foro \\
\hline Dientes anteriores & ayewe foro \\
\hline Embarazada & niepüñeñün, niechelen \\
\hline Encía & ürüm \\
\hline Enfermedades crónicas & kutran \\
\hline Estética & adngechi \\
\hline Exodoncia & nentuforon \\
\hline Fractura dental & watroy foro \\
\hline Halitosis & nümuforo /nümüwün \\
\hline Higiene & liftuwün \\
\hline Hueso & foro \\
\hline Incisivo & ayewe \\
\hline Inflamación & küfün \\
\hline Labios & mellfü wün \\
\hline Lengua & kewün \\
\hline Mandíbula & Tranga-tranga \\
\hline Masticación & trangatrangatun, küchawün \\
\hline Medicamentos & Wingka lawen \\
\hline Mejilla & ange \\
\hline Molar & ülnga \\
\hline Movilidad dentaria & Rupaley foro \\
\hline Músculos & fün \\
\hline Necrosis pulpar & Lay fün \\
\hline Nervio & Fün foro \\
\hline Paladar duro & fidafida \\
\hline Pulpa dental & Fün foro \\
\hline Saliva & ko wün \\
\hline Sangramiento & mollfütun \\
\hline Sensibilidad & pichikutran \\
\hline Supuración & trarün \\
\hline Tratamiento & Ina lawen \\
\hline Tercer molar & lüngerma foro \\
\hline Urgencia & matun \\
\hline
\end{tabular}

Tabla II. Listado de términos clínico-odontológicos sin traducción literal, pero puede comprenderse el concepto desde la lengua.

\begin{tabular}{lll}
\hline Abfracción & Enfermedad periodontal & Premolar \\
Alveólos & Fístula & Rehabilitación \\
Anestesia & Fisura & Resto radicular \\
Bruxismo & Hemorragia & Tártaro \\
Cepillo de dientes & Limpieza dental & Tinción dentaria \\
Contacto oclusal & Paladar blando & \\
Destartraje & Periodontitis & \\
\hline
\end{tabular}

$56,81 \%$ (75) no existe traducción literal ni puede entenderse desde la lengua, por lo que se debe recurrir al préstamo castellano o a la creación de una nueva palabra (Tabla III). Para el análisis estadístico se utilizó la prueba no paramétrica chi cuadrado de Pearson, la cual determinó que existen diferencias significativas entre las proporciones de los tres grupos de términos estudiados $(p=0,000)$.

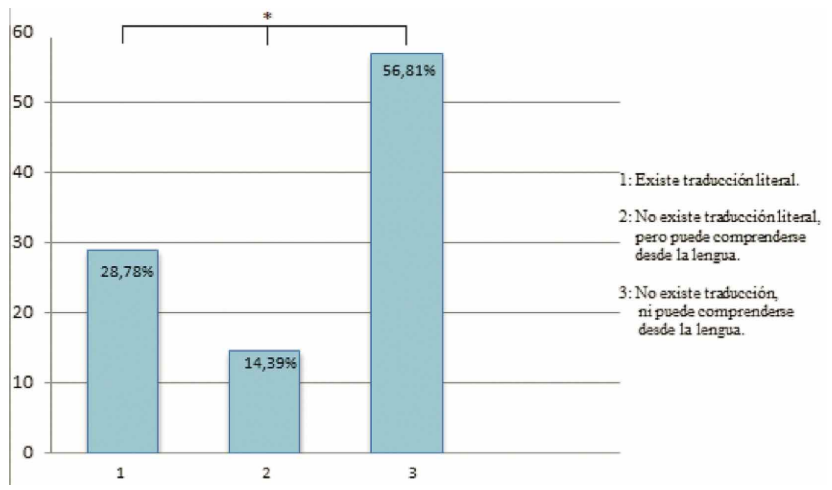

Fig. 1. Distribución de términos odontológicos según su traducción al mapuchedungun.

\section{DISCUSIÓN}

Para algunas comunidades indígenas timote de Venezuela, las enfermedades de la cavidad oral se centran en la caries dental (picadura o herida del diente) y la enfermedad periodontal (encías reventadas), otorgando poca o nula relevancia a las pérdidas dentarias, con o sin compromisos estéticos o funcionales, maloclusiones dentarias u otras enfermedades oral de tejidos blandos y duros. (Reinaldo \& Zambrano, 2009). En el caso del pueblo mapuche, la concepción acerca de la salud oral pareciera tener mucha importancia, lo que puede ser observado en como la lengua describe los dientes, por ejemplo:

\author{
Aye-we \\ Sonreir-INSTRUMENTO \\ Instrumento para la sonrisa \\ "Incisivo"
}

El ejemplo anterior da cuenta de una concepción relativa a la función de la anatomía dentaria, por lo que es muy habitual que la mayoría de los ancianos mapuches, cuando han perdido sus dientes, tiendan a reemplazarlos por medio de aparatos protésicos. 
Tabla III. Listado de términos clínico-odontológicos sin traducción literal, ni puede comprenderse desde la lengua.

\begin{tabular}{lll}
\hline Abceso & Dolor espontáneo & Placa bacteriana \\
Abfracción & Dolor mantenido & Pulpectomía \\
Aguja & Dolor noctumo & Pulpitis \\
Alergia & Dolor pulsatil & Pulpotomía \\
Alvéolo & Dolor provocado & Profilaxis \\
Analgésicos & Erosión & Provisorio \\
Antibióticos & Esmalte dental & Protesis fija- coronas \\
Antiinflamatorios & Frenillos labiales & Prótesis removible- placas \\
Apicectomía & Fresa & Pulido coronario \\
Articulación temporomandibular & Fluoración & Pulido radicular \\
Aumento de volumen & Ganglio sano & Quiste \\
Bacterias & Ganglio inflamado & Radiografía \\
Bruxismo & Gingivitis & Raices dentarias \\
Cavidad de caries & Granuloma & Rebordes óseos \\
Cirugía & Glándulas salivales & Remineralización \\
Canal radicular & Hipertensión & Reconstitución de resina \\
Cuello del diente & Implantes & Reconstitución de ionómero \\
Cubeta & Impresión & Reconstitución de amalgama \\
Dentina & Incrustación & Retracción gingival \\
Desmineralización & Infección & Seda dental \\
Diabetes & Maxilar & Sellante \\
Dolor agudo & Modelos de yeso & Sensibilidad post operatoria \\
Dolor articular & Operatoria & Sutura \\
Dolor al calor & Pasta dental & Tratamiento periodontal \\
Dolor al frío & Piso de cavidad oral & Tratamiento de endodoncia \\
Dolor a la percusión & Oseointegracion & Vestíbulo \\
\hline
\end{tabular}

Entre las comunidades hablantes del mapuchedungun y sus tratantes de habla castellana, se genera una brecha comunicacional importante desde el punto de vista odontológico, al no contar con las herramientas lingüísticas necesarias que permitan al clínico expresar indicaciones específicas que sean efectivamente comprendidas por sus pacientes, como en el caso del diagnóstico y etiología de los cuadros que les afectan odontológicamente, ocasionando que la comunicación se torne muy difícil entre paciente y tratante. Aún más, este déficit en las herramientas comunicacionales de la lengua hablada, tiene relación con elementos de descripción del dolor, anatomía, patología y tratamiento, limitando la integración de conceptos indispensables para la resolución de enfermedades orales y la recuperación de la salud oral. En situaciones como la descrita, se hace necesaria la creación e incorporación de nuevos vocablos, lo que constituye el enriquecimiento de una lengua y a la vez un reconocimiento y valoración de la lengua minorizada. Esta tarea se realiza a través de la creación de neologismos. La neología se define como la disciplina que estudia los fenómenos nuevos que aparecen en las lenguas, los que pueden manifestarse en cualquiera de sus niveles descriptivos: fonético, fonológico, morfológico, sintáctico y léxico. Cuando estos fenómenos se representan en el último de estos planos, hablamos de neología léxica (Villena Araya, 2010). Para el caso de la lengua mapuche, en estos últimos treinta años, especialmente en el ámbito educativo, se han generado una serie de neologismos vinculados a educación, por ejemplo: chillkatuwe 'escuela', kimeltuchefe 'profesor', wiriwe 'lapiz', trawupeyüm 'sala de clases', entre otros. Por su carácter aglutinante, la mayoría de los neologismos que se han creado en la lengua, son composiciones de más de un elemento, tal como se observa en el ejemplo 1:

$$
\begin{gathered}
\text { Kim-el-tu-che-fe } \\
\text { Saber-BEN-VERB-persona-AGEN } \\
\text { "Persona que enseña" } \\
\text { Profesor }
\end{gathered}
$$

Si observamos el ejemplo 1, se aprecia como la lengua suma distintos elementos: la acción (verbos), agentes de acción (personas), describiendo un rol, de manera que la aglutinación de varios sufijos descripti- 
vos, generan una sola idea. En el caso de la terminología odontológica, el idioma mapuche tiene un amplio repertorio para identificar una importante cantidad de conceptos vinculados a dientes, elementos y partes de la boca, enfermedades, entre otros aspectos. En casos de ausencia de la palabra se propone la creación de nuevos términos siguiendo el proceso anteriormente descrito, o bien, recurrir al uso de préstamos de otras lenguas cuando no sea posible o necesario crear una nueva palabra. El ejemplo 2, nos ilustra una posibilidad para 'cepillo de dientes':

\section{Kücha-foro-peyüm \\ Lavar-diente-INSTRUMENTO \\ "cepillo de dientes"}

\section{CONCLUSIÓN}

Por medio de este estudio concluimos que existe un porcentaje importante de términos, usados frecuentemente en la atención odontológica (56,81\%), que no tienen traducción en mapuchedungun; y aunque entre el pueblo mapuche la concepción acerca de la salud oral pareciera tener mucha importancia, aun son escasos los términos clínicos que se pueden explicar en base a conceptos mapuches preexistentes. Por tanto, ante la necesidad de generar vías de comunicación certera entre el paciente y tratante, así como, de contribuir al mejoramiento de la atención odontológica que el paciente recibe, sería recomendable crear nuevas palabras y conceptos a través del uso de préstamos lingüísticos y neologismos (Chiodi \& Loncón, 1999), con el objeto de ampliar el vocabulario odontológico en mapuchedungun respetando la cosmovisión del pueblo mapuche, con la ayuda de loncos, ancianos, líderes de comunidades y especialistas en dicha lengua.

FLORES, T.; CANIGUAN J. \& FUENTES, R. Analysis of clinical dental terminology and its translation to Mapudungun. Int. J. Odontostomat., 10(2):249-254, 2016.

ABSTRACT: Western medicine use has an important role in the research of quality communication with the Mapuche ethnicity, which has been effective through the creation and implementation of Intercultural health clinics. However, language gaps are still a great communication problem. Bilingual Mapudungun-Castilian dictionaries give limited information regarding the necessary terms for a correct diagnosis in dentistry. The same issue is true for translation terms for some concepts with regard to the symptoms and signs of oral pathologies. The objective of this research was to determine whether the terms used in dentistry have a Mapudungun translation. A qualitative, descriptive and crosssectional study was performed. With the help of 10 dentists in public health practice in Temuco, we developed a list of 132 terms frequently used in dental practice. Its significance was discussed with the help of Mapudungun-Castilian dictionary and specialists in the Mapuche language. It was determined that $28.78 \%$ of the terms have a Mapuche language translation; $14.39 \%$ have no translation but can be understood from the Mapudungun language; while 56.81 $\%$ have no translation and can't be understood from the Mapudungun language. For the statistical analysis, Pearson Chi square non-parametric test was used in order to determine significant differences between the proportions of the studied terms from the groups $(p=0,000)$. A significant number of terms commonly used in dentistry don't have Mapudungun translation, even though among the Mapuche people the concept of oral health appears to be very important. There are still few clinical terms that can be explained on the basis of pre-existing Mapuche concepts. It is necessary to generate accurate forms of communication between the patient and the dentist, along with the creation of new words and concepts in Mapudungun, and therefore tools that might help to understand the perception of concepts of health and disease in the Mapuche worldview context to improve dental care. natives.

KEY WORDS: dentist, Mapuche, belief, oral health,

\section{REFERENCIAS BIBLIOGRÁFICAS}

Antinao Varas, C. Diccionario Ta in Mapun Dungun. Nuestra Lengua Mapuche. Santiago de Chile, Pu Lifru Mapunche kimün ngelu, 2014.

Augusta, F. J. Diccionario Mapuche-Español. Tomos I y II. $2^{\text {a }}$ ed. Santiago de Chile, Ediciones Seneca, 1995.

Augusta, F. J. Diccionario Araucano: Mapuche-Español, Español-Mapuche. Santiago de Chile, Ediciones Cerro Manquehue, 2007

Centro de Estudios Públicos (CEP). Una Radiografía de los Mapuches. Estudio Nacional de Opinión Pública No 43 del Centro de Estudios Públicos. Santiago de Chile, Centro de Estudios Públicos, 2002

Chiodi, F. \& Loncón, E. Crear Nuevas Palabras: Innovación y Expansión de los Recursos Lexicales del Mapudungun. Temuco, Instituto de Estudios Indígenas, Universidad de la Frontera, 1999.

Departamento de Estudios y Desarrollo de la División de Planificación y Presupuesto del Ministerio de Educación. 
FLORES, T.; CANIGUAN, J. \& FUENTES, R. Análisis de terminología clínico odontológica y su traducción al mapudungun. Int. J. Odontostomat., 10(2):249-254, 2016.

Indicadores de la Educación en Chile / Año 2002. Santiago de Chile, Ministerio de Educación, República de Chile, 2004. Disponible en: http://www.oei.es/quipu/chile/Indicadores_Educ2002.pdf

Díaz Mujica, A.; Pérez Villalobos, M. V.; González Parra, C. \& Simon, J. W. Conceptos de enfermedad y sanación en la cosmovisión mapuche e impacto de la cultura occidental. Cienc. Enferm., 10(1):9-16, 2004.

Errázuriz, F. G. El pueblo Mapuche: Historia, medicina y proyectos de coexistencia en el área de la salud (Primera parte). Rev. Chil. Pediatr., 77(3):290-4, 2006.

Haughney, D. \& Marimán, P. Población Mapuche: Cifras y Criterios. Documento de Trabajo No 1. Liwen, Centro de Documentación Mapuche, Ñuke Mapu, 1993. Disponible en: http://www.mapuche.info/mapuint/liwdoc1a.htm

Ibacache, J. Rume kgen mew ta az mapu / Epidemiología de la Transgresión en Maquehue-Pelale. Temuco, Unidad de salud con Pueblos Indígenas, Servicio de Salud Araucanía Sur, 2001.

Marileo, A. Mundo Mapuche. Santiago de Chile, Meridión Comunicaciones, 2002.

Ministerio de Salud (MINSAL). Foros de Salud: Desafíos y propuestas ciudadanas. Resultados del Proceso Foros: "Construyamos Juntos una Mejor Salud para Todos y Todas al 2020". Santiago de Chile, Equidad.cl, Secretaría de Determinantes Sociales de la Salud, Ministerio de Salud, Gobierno de Chile, 2010. Disponible en: http:// www.yafun.cl/descargas/foros-nacional-baja.pdf

Reinaldo, E. \& Zambrano, V. La familia indígena timote y sus prácticas de salud bucal. Costumbres, creencias y valoraciones. Fermentum Rev. Venez. Sociol. Antropol., 19(56):518-29, 2009.

Villena Araya, B. Creación neológica en mapudungún: entre el desplazamiento y la lealtad lingüística. Terminàlia, (10):37-49, 2014.
Dirección para Correspondencia:

Prof. Dr. Ramón Fuentes Fernández

Centro de Investigación en Ciencias Odontológicas

(CICO)

Facultad de Odontología

Universidad de La Frontera

Temuco

CHILE

Email: ramón.fuentes@ufrontera.cl

Recibido : 12-08-2015

Aceptado: 29-06-2016 